\title{
Enhanced 5-methylcytosine detection in single- molecule, real-time sequencing via Tet1 oxidation
}

\author{
Tyson A Clark', Xingyu Lu², Khai Luong', Qing Dai ${ }^{2}$, Matthew Boitano ${ }^{1}$, Stephen W Turner', Chuan $\mathrm{He}^{2}$ and \\ Jonas Korlach ${ }^{1 *}$
}

\begin{abstract}
Background: DNA methylation serves as an important epigenetic mark in both eukaryotic and prokaryotic organisms. In eukaryotes, the most common epigenetic mark is 5 -methylcytosine, whereas prokaryotes can have 6-methyladenine, 4-methylcytosine, or 5-methylcytosine. Single-molecule, real-time sequencing is capable of directly detecting all three types of modified bases. However, the kinetic signature of 5-methylcytosine is subtle, which presents a challenge for detection. We investigated whether conversion of 5-methylcytosine to 5-carboxylcytosine using the enzyme Tet1 would enhance the kinetic signature, thereby improving detection.

Results: We characterized the kinetic signatures of various cytosine modifications, demonstrating that 5-carboxylcytosine has a larger impact on the local polymerase rate than 5-methylcytosine. Using Tet1-mediated conversion, we show improved detection of 5-methylcytosine using in vitro methylated templates and apply the method to the characterization of 5-methylcytosine sites in the genomes of Escherichia coli MG1655 and Bacillus halodurans C-125.

Conclusions: We have developed a method for the enhancement of directly detecting 5-methylcytosine during single-molecule, real-time sequencing. Using Tet1 to convert 5-methylcytosine to 5-carboxylcytosine improves the detection rate of this important epigenetic marker, thereby complementing the set of readily detectable microbial base modifications, and enhancing the ability to interrogate eukaryotic epigenetic markers.
\end{abstract}

Keywords: Carboxylcytosine, DNA sequencing, epigenomics, methylation, methylcytosine, SMRT sequencing, Tet protein

\section{Background}

The DNA of most organisms is comprised of more than the four canonical bases (A, C, G and T). In mammals, for example, 5 -methylcytosine $(5 \mathrm{mC})$ constitutes about $1 \%$ of all DNA bases and is found primarily in CpG dinucleotides. Methylation plays a critical role in the regulation of gene expression, genomic imprinting and the suppression of transposable elements [1]. Often referred to as the sixth base, 5 -hydroxymethylcytosine $(5 \mathrm{hmC})$ is also found in many metazoan genomes [2]. $5 \mathrm{hmC}$ is converted from $5 \mathrm{mC}$ by the Ten-eleven translocation (Tet) family of proteins $[3,4]$. Recently, it was discovered that Tet proteins can also convert $5 \mathrm{mC}$ to 5 -formylcytosine (5fC) [5] and 5carboxylcytosine $(5 \mathrm{caC})[6,7]$. In humans, there are three

\footnotetext{
* Correspondence: jkorlach@pacificbiosciences.com

'Pacific Biosciences, 1380 Willow Road, Menlo Park, CA 94025, USA

Full list of author information is available at the end of the article
}

different Tet proteins (Tet1, Tet2, Tet3) that are all capable of this conversion [6,7]. It is currently thought that DNA demethylation may occur through this process of $5 \mathrm{mC}$ oxidation followed by base excision repair $[6,8]$, and possibly decarboxylation [9].

Many of the genomes of bacteria and archea also contain modified DNA bases [10]. The three most common forms of methylation are 6-methyladenine $(6 \mathrm{~mA})$, 4-methylcytosine $(4 \mathrm{mC})$ and $5 \mathrm{mC}$. The primary function of methylation is DNA self-recognition via restrictionmodification systems that protect the organism against invading DNA. However, there are methyltransferases (MTases), such as dam, that do not fall in restrictionmodification systems and are important in chromosome stability, mismatch repair and replication [11]. There is some evidence that the presence of methylation can also impact gene expression [12]. Thus, detection and
C Biomed Central

C 2013 Clark et al; licensee BioMed Central Ltd. This is an Open Access article distributed under the terms of the Creative Commons Attribution License (http://creativecommons.org/licenses/by/2.0), which permits unrestricted use, distribution, and reproduction in any medium, provided the original work is properly cited. 
identification of methylated bases in both prokaryotes and eukaryotes is essential to the complete understanding of genome function.

The most common techniques for large-scale detection of DNA methylation rely on bisulfite treatment of the DNA prior to sequencing. Sodium bisulfite chemically deaminates cytosine residues to uracil, which are subsequently read out as thymine. Methylated cytosines are converted with much lower efficiency and thus remain cytosines. The presence of $5 \mathrm{mC}$ is inferred from comparing bisulfite-treated DNA sequences to an untreated reference. In standard bisulfite sequencing, $5 \mathrm{mC}$ cannot be distinguished from $5 \mathrm{hmC}$ [13]. The conversion of $5 \mathrm{mC}$ to $5 \mathrm{caC}$ through the activity of Tet 1 [14] and $5 \mathrm{hmC}$ to $5 \mathrm{fC}$ through chemical conversion [15] followed by bisulfite sequencing runs has recently been exploited for the genome-wide sequencing of $5 \mathrm{mC}$ and $5 \mathrm{hmC}$.

We have previously described a technique for the direct detection of modified DNA using single-molecule, realtime $\left(\mathrm{SMRT}^{\circledR}\right)$ sequencing $[16,17]$. SMRT sequencing involves the monitoring of a DNA polymerase as it makes a copy of a DNA molecule $[18,19]$. When the DNA polymerase encounters a modified base on the template strand, its rate of progression changes in a characteristic way relative to an unmodified template with the same sequence context $[16,17]$. The speed of the polymerase is monitored by determining the length of time between the fluorescent pulses that indicate nucleotide incorporation. The time between pulses is called the interpulse duration (IPD). The change in IPD between a modified and control template varies in magnitude and position depending on the nature of the base modification and the local sequence context. We refer to these reproducible changes in IPD as the kinetic signature for that modification.

Although many base modifications, such as $6 \mathrm{~mA}, 4 \mathrm{mC}$, $5 \mathrm{hmC}$ and 8-oxo-guanine, are readily detectable in SMRT sequencing $[16,17,20,21]$, the kinetic signature of $5 \mathrm{mC}$ is more subtle, requiring high sequencing fold coverage to make out the small effect on polymerase speed. The methyl group is small, and unlike for the case of $6 \mathrm{~mA}$ and $4 \mathrm{mC}$, it is oriented towards the major groove and is not involved in base pairing - in fact the methyl group has to be readily accepted by DNA polymerases at this position as it is present on thymine, the other canonical pyrimidine base. We hypothesized that conversion of $5 \mathrm{mC}$ into a larger group may increase the magnitude of the kinetic signature during SMRT sequencing, thus enhancing the ability to detect $5 \mathrm{mC}$. The Tet family of proteins carries out conversion of $5 \mathrm{mC}$ to several other modified forms of cytosine including $5 \mathrm{hmC}, 5 \mathrm{fC}$ and $5 \mathrm{caC}[6,7]$. This strategy has been shown to be effective in the recently developed Tet-assisted bisulfite sequencing of $5 \mathrm{hmC}$ [14].
Here, we demonstrate that mouse Tet1 (mTet1) can be used to enhance direct detection of $5 \mathrm{mC}$ during SMRT sequencing. Using synthetic templates made from oligonucleotides containing $5 \mathrm{mC}, 5 \mathrm{hmC}, 5 \mathrm{fC}$ or $5 \mathrm{caC}$ modifications, we tested the kinetic signatures of each modification. We discovered that each of the moieties into which $5 \mathrm{mC}$ can be converted via Tet increased the magnitude of the kinetic signature, with $5 \mathrm{caC}$ having the largest effect. Next, we observed that oxidation of $5 \mathrm{mC}$ to $5 \mathrm{caC}$ on either synthetic templates or in vitro methylated DNA enhanced our ability to detect positions of $5 \mathrm{mC}$. We then used our improved $5 \mathrm{mC}$ detection method for the genome-wide characterization of MTase activities in two different bacterial strains.

\section{Results}

\section{SMRT sequencing shows varying kinetic signatures for} different cytosine modifications

To determine the kinetic signatures for the four naturally occurring forms of cytosine with a modification on the fifth carbon atom, we designed synthetic SMRTbell templates made from oligonucleotides with modified cytosines at specific template positions. Four modified synthetic SMRTbell templates were made, each containing two $5 \mathrm{mC}, 5 \mathrm{hmC}, 5 \mathrm{fC}$ or $5 \mathrm{caC}$ modifications. The polymerase dynamics of each was analyzed by SMRT sequencing and compared with a control template of the same sequence but lacking the modifications. The kinetic signatures for each cytosine modification type are shown in Figure 1 as ratios of the average IPD value at each template position of the modified template relative to the unmodified control. The positions of the modified bases are highlighted as red bars. As observed previously [17], the kinetic signature for $5 \mathrm{mC}$ is distinct from the background, but the magnitudes of the IPD ratios are small, translating to relatively high sequencing coverage for detection of the modified positions with high confidence. Furthermore, the kinetic signature is spread out over multiple positions on the DNA template [17], likely due to effects of base modifications on the polymerization rate extending across the entire footprint of the polymerase [22].

As the size of the chemical structure of the modification increases, the magnitude of the kinetic signature also increases. The IPD ratio peaks range from approximately two-fold for $5 \mathrm{mC}$ and approximately three-fold to higher than five-fold for $5 \mathrm{fC}$ and $5 \mathrm{caC}$ (Figure 1). For each modification type, an extended signature consisting of multiple IPD ratio peaks was observed, with the most prominent signals at positions $0,+2$ and +6 relative to the polymerase movement, with 0 being the position of the modification in the template. In most instances investigated here, the +2 peak was the most pronounced. As previously observed $[16,17]$, the kinetic signatures for a given modification 

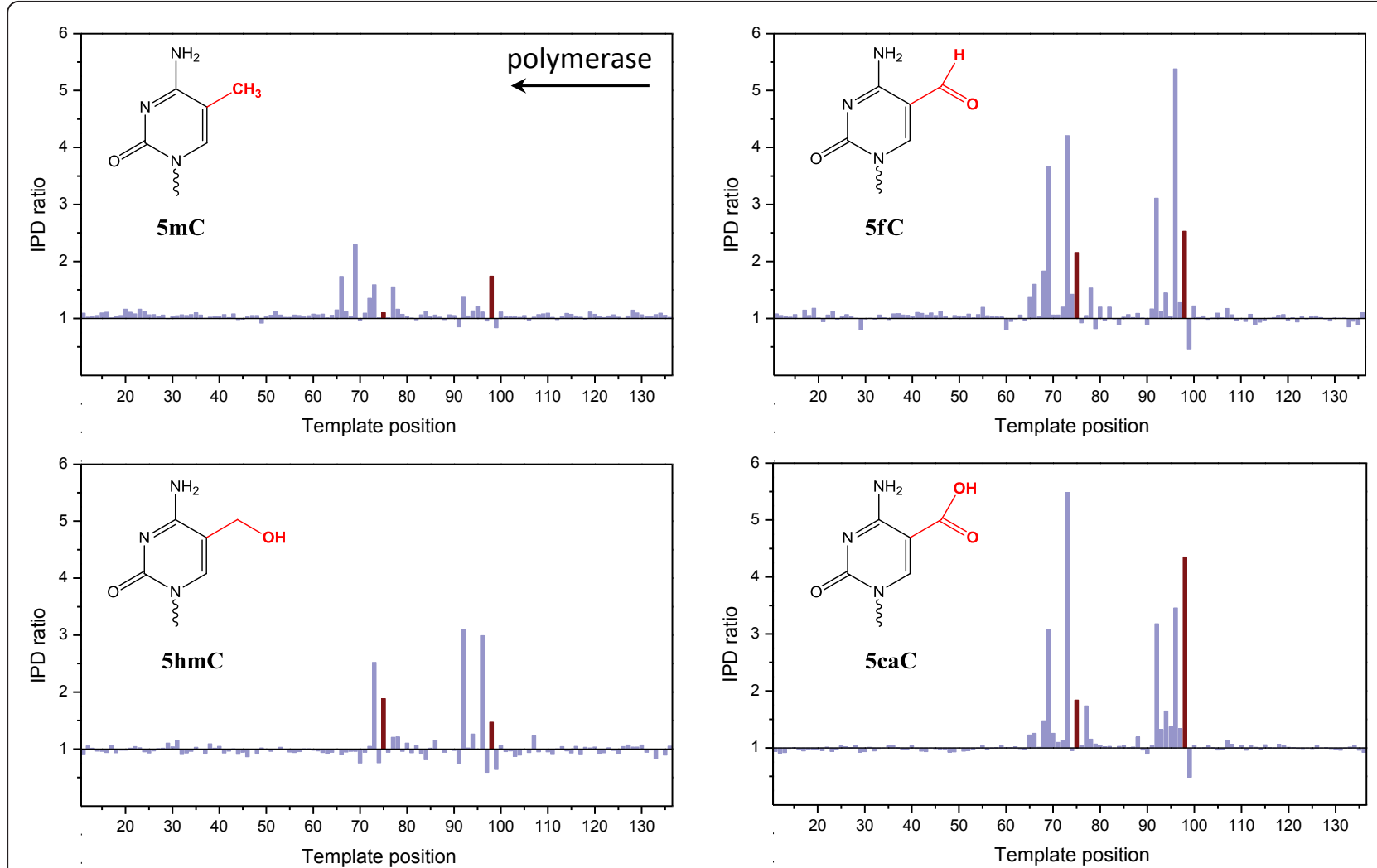

Figure 1 Kinetic signals from SMRT sequencing for the four epigenetic markers of cytosine $5 \mathrm{mC}, 5 \mathrm{hmC}, 5 \mathrm{fC}$ and $5 \mathrm{caC}$. Synthetic oligonucleotides carrying two modified sites each (red bars) were subjected to SMRT sequencing and the polymerase kinetics compared by plotting the ratio of IPDs for each template position against a control template of identical sequence but lacking the modifications. The template is shown in the $5^{\prime}$ to $3^{\prime}$ direction from left to right, the polymerase movement is right to left across the template as indicated by the arrow. 5caC: 5-carboxylcytosine; 5fC: 5-formylcytosine; 5hmC: 5-hydroxymethylcytosine; 5mC: 5-methylcytosine; IPD: interpulse duration; SMRT: single-molecule: real-time.

varied slightly depending on the surrounding sequence context. These differences in the pattern and magnitudes of the kinetic signatures for each of the four different modifications are a parameter that can be used to discriminate between different modifications on the same DNA template, although they are not used in the current implementation of the software. To further explore the effects of local sequence context on the kinetic signatures of $5 \mathrm{mC}$ and $5 \mathrm{caC}$, we used a synthetic SMRTbell template that contained a modified base in a 5'-CG-3' sequence context, surrounded by two random bases on each side. Additional file 1 shows a heat map of IPD ratios for the 256 possible sequence contexts at each position from -3 to +6 relative to the modified position in the template. As observed previously $[17,20]$, the magnitude and position of the kinetic signals for both $5 \mathrm{mC}$ and $5 \mathrm{caC}$ are dependent upon the surrounding sequence context. The conversion of $5 \mathrm{mC}$ to $5 \mathrm{caC}$ enhances the magnitude of the IPD ratio at each position where ratios above 1.0 are observed for $5 \mathrm{mC}$, that is, positions $0,+2$, and +6 , and brings out an additional detectable signal at the -2 position for some sequence contexts. Tet conversion enhances the kinetic signals relatively evenly across all sequence contexts, which is apparent from the good preservation of the overall sequence context profiles. We are currently investigating possible additional correlations that could exist between different base positions in a given context. This could aid in the development of more refined identification algorithms.

\section{Enhanced detection of $5 \mathrm{mC}$ following conversion to $5 \mathrm{caC}$ by $\mathrm{mTet} 1$}

Because $5 \mathrm{caC}$ has the largest kinetic signature, conversion of $5 \mathrm{mC}$ to $5 \mathrm{caC}$ should significantly improve the ability to detect $5 \mathrm{mC}$ in SMRT sequencing. The Tet family of proteins has been shown to convert $5 \mathrm{mC}$ to $5 \mathrm{caC}$ in mammalian genomes [6,7]. This conversion can be over $97 \%$ for sequencing purposes and does not exhibit significant sequence context bias [14]. We tested the ability of Tet1-mediated oxidation of $5 \mathrm{mC}$ to $5 \mathrm{caC}$ to enhance direct detection on in vitro methylated DNA templates, described in detail in Methods. Briefly, we first generated an approximately 6 -kb plasmid by inserting a 
lambda DNA fragment into the pCRBlunt vector and subjected it to whole genome amplification (WGA) to erase all modifications. We then generated an approximately 500 bp randomly sheared shotgun SMRTbell template library from the WGA material, followed by in vitro methylation using the HpaII MTase that modifies the internal cytosine in 5'-CCGG-3' sequence contexts. Considering both the forward and reverse DNA strands, the plasmid sequence contains 70 instances of the 5'CCGG-3' sequence motif. Methylated positions within the SMRTbell templates were converted to $5 \mathrm{caC}$ by treatment with the Tet 1 enzyme. In vitro methylated $(5 \mathrm{mC})$, Tet1 converted $(5 \mathrm{caC})$ and WGA control (no modification) libraries were then subjected to SMRT sequencing.

Figure 2 shows the plasmid-wide view of IPD ratio data for the in vitro methylated (Figure 2a) and the mTet1-converted (Figure 2b) templates relative to the unmodified control. The IPD ratios for the $5 \mathrm{mC}$-modified templates are visible as small excursions from the background (Figure 2a). Following mTet1-mediated oxidation to $5 \mathrm{caC}$ (Figure 2b), the kinetic signature was enhanced by an average of approximately 4.6 -fold, making all 35 instances of the MTase recognition motif recognizable as large excursions in the IPD ratio. The primary IPD ratio peaks for the $5 \mathrm{caC}$ sample again fell at the +2 position relative to the modification, consistent with the results obtained from the synthetically derived samples. Similar results were obtained with synthetic SMRTbell templates that were made with oligonucleotides containing $5 \mathrm{mC}$ modifications and subjected to conversion by mTet1 (Additional file 2).

\section{Genome-wide analysis of 5mC DNA methyltransferase specificities in bacterial strains}

Most bacterial and archeal genomes contain DNA MTases. Many of these MTases are paired with restriction endonucleases as part of a restriction-modification system that protects the organism from foreign DNA [23]. These MTases typically methylate a specific sequence context, which blocks the activity of the restriction enzyme that recognizes the same site. The three most common types of methylation found in bacteria and archea are $6 \mathrm{~mA}, 4 \mathrm{mC}$ and $5 \mathrm{mC}$. To test the ability of the $\mathrm{mTet} 1$-enhanced signal to detect $5 \mathrm{mC}$ in genomic DNA, we selected two bacterial strains that are known to express a $5 \mathrm{mC}$ MTase [24].

Escherichia coli K12 MG1655 is a well-studied, common laboratory strain that is known to express three different MTases. EcoKdam is a $6 \mathrm{~mA}$ MTase that modifies the adenosine in a 5'-GATC-3' sequence context (methylated base underlined). EcoKI is a type I MTase that modifies the sequence context 5'-GCAC(N6)GTT3' and reverse complement 5'-AAC(N6)GTGC-3'. The $5 \mathrm{mC}$ MTase is EcoKdcm that modifies the internal cytosine in a 5'-CCWGG-3', where W is either an A or a $\mathrm{T}$. We made SMRTbell templates from randomly sheared E. coli K12 MG1655 genomic DNA, a portion of which was sequenced in its native form and another portion of which was subjected to the mTet1 treatment. Both samples were sequenced to approximately $150 \times$ per-DNA strand fold coverage.

We carried out an unbiased search for sequence motifs that were enriched in proximity to genomic positions with large excursions from the expected IPD values (see Methods for details). For the native sample we identified the expected 5'-GATC-3' and the 5'-GCAC(N6)GTT-3' and/or 5'-AAC(N) 6 GTGC-3' sequence motifs, but observed low signal levels for the 5'-CCWGG-3' motif. However, following the mTet 1 conversion, we were able to identify the majority of 5'-CCWGG-3' motifs in the genome as modified. Figure 3 compares IPD ratio data over the entire $E$. coli genome before and after mTet 1 treatment. As expected, IPD ratio data for sites methylated with m6A did not change between the native and Tet1-converted samples (panel a, grey lines). By contrast, IPD ratio data for the +2 position of the 5'-CWGG-3' sites (panel a, red lines) were significantly increased in the mTet1-treated sample, thereby improving detection of $d c m$-mediated methylated positions, with IPD ratio magnitudes now similar to m6A signals. The distributions of IPD ratios for all methylated motifs are included in Additional file 3.

To estimate the degree of enhancement in $5 \mathrm{mC}$ detection by mTet1 treatment (Table 1), we selected the $99^{\text {th }}$ percentile kinetic score of an off-target motif (5'GGWCC-3') as the threshold for calling a genomic position as methylated (Figure 3c). Any kinetic score that was greater than this value was considered modified. In the native sample, only 455 (1.9\%) of all genomic 5'-CCWGG3 ' positions were detected above this background value. Upon conversion of $5 \mathrm{mC}$ to $5 \mathrm{caC}$ in the mTet1-treated sample, 22,913 genomic 5'-CCWGG-3' positions (95.2\%) were detected as methylated. The off-target site was unaffected by the mTet 1 treatment, highlighting the specificity of the mTet1 conversion to methylated DNA sites. Additional file 4 shows the detection rate for all modified sequence motifs, including $6 \mathrm{~mA}$. This table also enumerates detection levels of additional off-target sequences that exhibit a consistently low percentage of sites above the detection threshold.

We performed the same procedure for B. halodurans $C-125$, a bacteriocin-producing soil bacterium. The $B$. halodurans genome is predicted to have three different MTases [24], including one MTase that has the hallmarks of a $5 \mathrm{mC}$-modifying enzyme. However, unlike for the E. coli sample, the exact sequence motifs and positions of the modifications are not known. Through SMRT sequencing, we were able to identify two methylated 


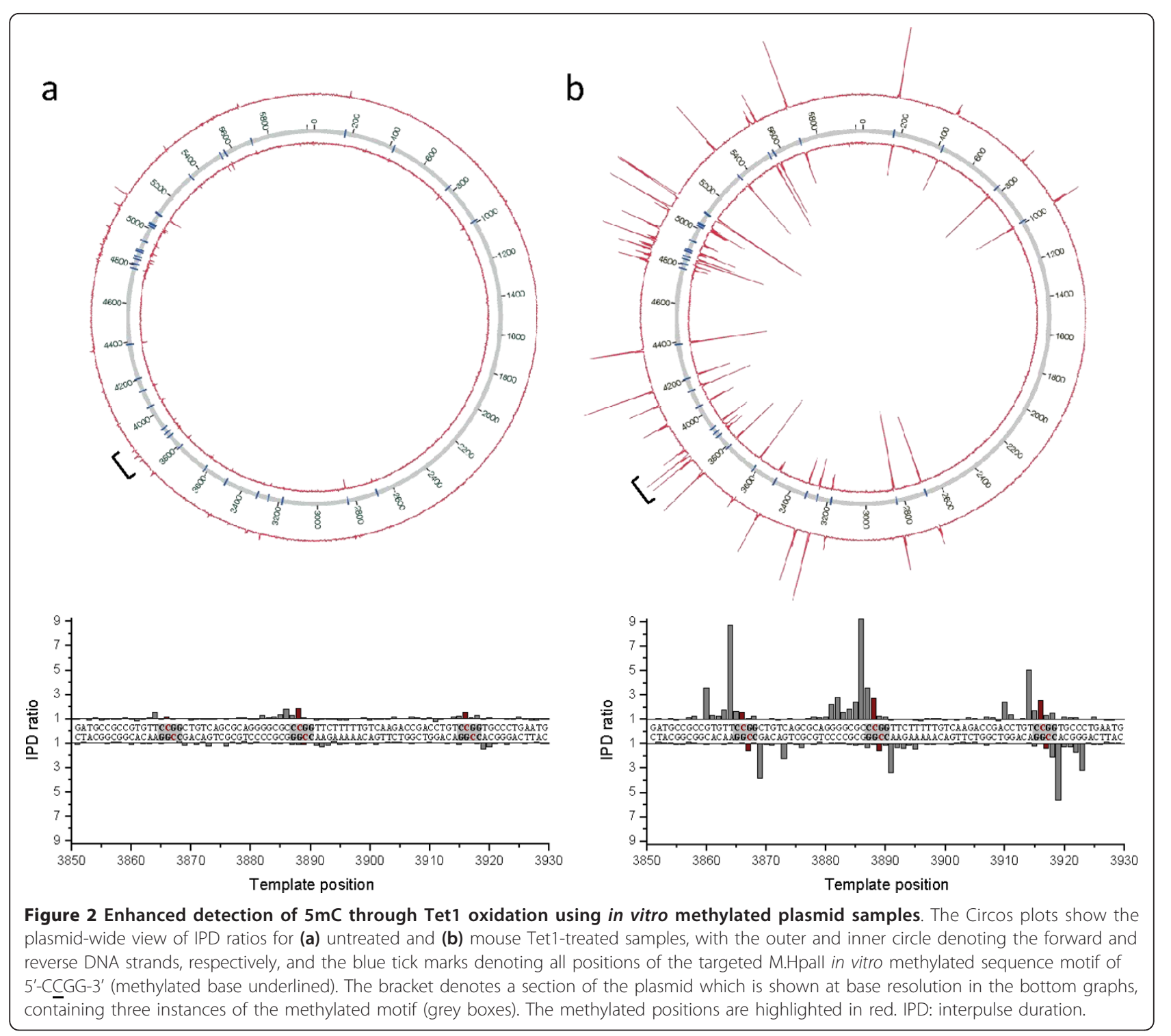

sequence motifs: 5'-GCATC-3' or 5'-GATGC-3' and 5'GGCC-3'. The first motif had high IPD ratio values on the A position on both forward and reverse strands, which is indicative of $6 \mathrm{~mA}$. This signal was present in both native and mTet1-treated samples (Figure 4). The 5'-GGCC-3' motif was considerably stronger in the mTet1-treated sample, with the strongest peak on the first $\mathrm{G}$ in the motif. Using the +2 pattern of the converted $5 \mathrm{caC}$ signature, the most likely modified base is the inner $\mathrm{C}$ in the 5'-GGCC-3' motif. We detected $4.3 \%$ of 5'GGCC-3' motifs without mTet1-treatment, increasing to $76.7 \%$ following the enhancement of the $5 \mathrm{mC}$ signal by mTet1 conversion (Table 1). The distributions of IPD ratios for all methylated motifs in the $B$. halodurans genome are shown in Additional file 3 and the detection rate data are presented in Additional file 5.

\section{Discussion}

In SMRT sequencing, modified bases in the DNA template are identified by the transient slowing of the DNA polymerase at and around the site of the modification. We previously demonstrated the detection of $5 \mathrm{mC}$ and $5 \mathrm{hmC}$ through such kinetic analysis [17]. Here, we extend the spectrum of detectable base modifications to the full complement of currently known modified forms of cytosine. Both $5 \mathrm{fC}$ and $5 \mathrm{caC}$ showed an increased interference with polymerase movement compared with $5 \mathrm{mC}$, resulting in stronger kinetic signals in SMRT sequencing. In addition to the increased size of the modification, the higher polarity of the formyl and carboxyl group could also contribute to the increased signal levels.

In this work, we describe improving the direct SMRT sequencing of $5 \mathrm{mC}$ via $\mathrm{mTet} 1$-mediated oxidation to 


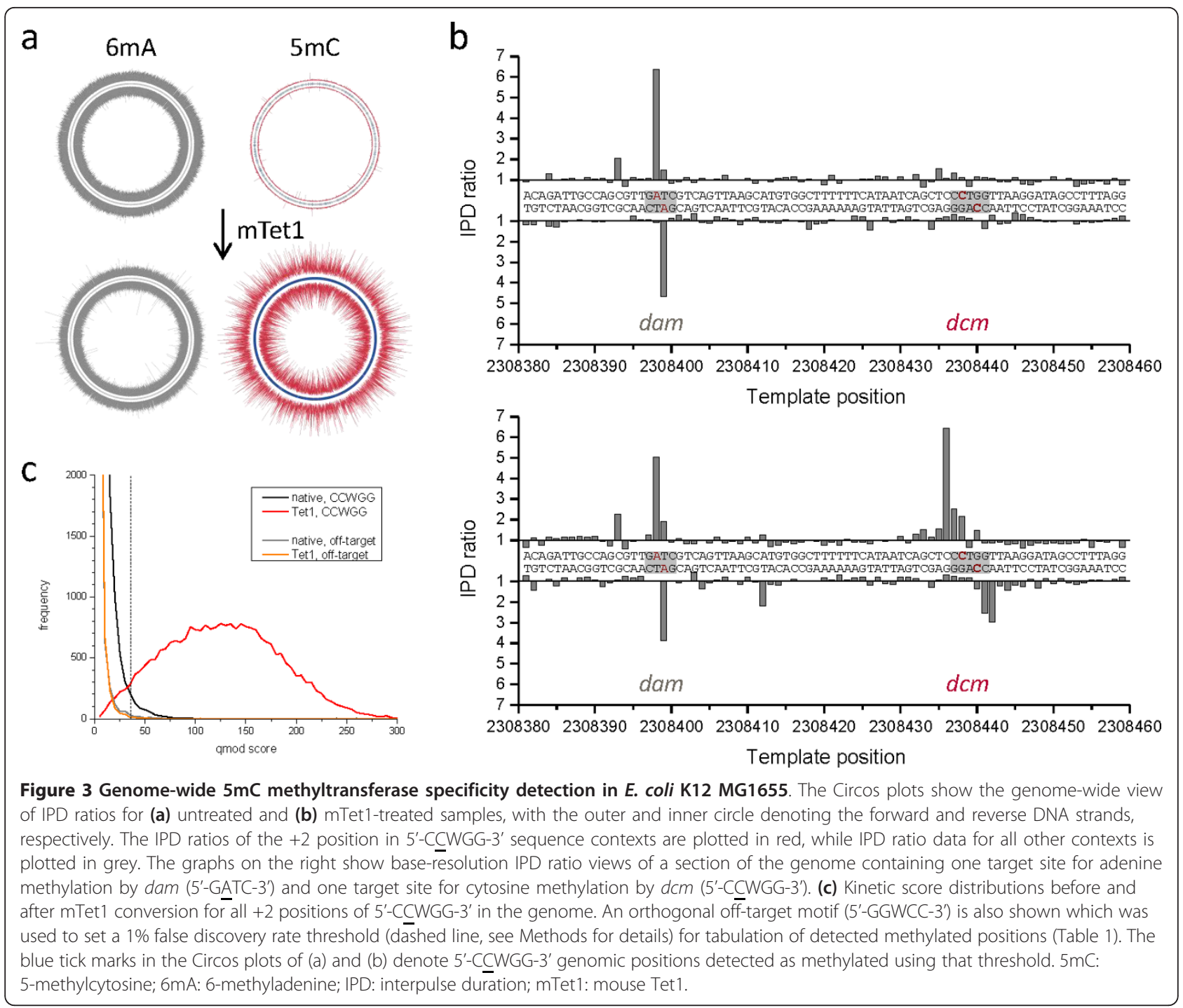

$5 \mathrm{caC}$, thereby reducing the relatively high sequencing coverage required to detect the subtle signals imparted by $5 \mathrm{mC}$ with high confidence. mTet 1 efficiently converted $5 \mathrm{mC}$ to $5 \mathrm{caC}$ in synthetic oligonucleotides, in vitro methylated plasmids, bacterial genomic DNA and mammalian genomic DNA [14], facilitating identification of microbial $5 \mathrm{mC}$ MTase specificities, thus complementing the other two common, readily detectable bacterial methylation marks of $\mathrm{m} 6 \mathrm{~A}$ and $\mathrm{m} 4 \mathrm{C}$ described previously [16,17]. The protocol is rapid and specific to $5 \mathrm{mC}$, allowing all three base modifications to be simultaneously detected in a single sequencing experiment. We anticipate that, for the sequencing of bacterial and archeal genomes, such comprehensive characterization of the methylome, in addition to de novo assembly of the genome [25,26], will improve our understanding of important microbiological phenomena, such as adaptation, pathogenicity and resistance evolution. It has been

Table 1 Detection of $5 \mathrm{mC}$ in native versus mTet1-enhanced SMRT sequencing for the bacterial genomes

\begin{tabular}{|c|c|c|c|c|c|c|}
\hline \multicolumn{2}{|c|}{ Sample } & \multirow{2}{*}{$\frac{\text { Methylation motif }}{C^{m} C W G G}$} & \multirow{2}{*}{$\frac{\text { Number in genome }}{24,079}$} & \multirow{2}{*}{$\frac{\text { Number detected }}{455}$} & \multirow{2}{*}{$\begin{array}{c}\text { Number detected (\%) } \\
1.9\end{array}$} & \multirow{2}{*}{$\begin{array}{c}\text { Number unassigned (\%) } \\
0.4\end{array}$} \\
\hline E. coli & native & & & & & \\
\hline MG1655 & Tet1 & $\mathrm{C}^{\mathrm{m}} \mathrm{CWGG}$ & 24,079 & 22,913 & 95.2 & 0.3 \\
\hline B. halodurans & native & $\mathrm{GG}^{\mathrm{m}} \mathrm{CC}$ & 15,207 & 660 & 4.3 & 0.6 \\
\hline C-125 & Tet1 & $\mathrm{GG}^{\mathrm{m}} \mathrm{CC}$ & 15,207 & 11,663 & 76.7 & 0.5 \\
\hline
\end{tabular}

\footnotetext{
${ }^{a}$ Unassigned is the percentage of genomic positions that have kinetic scores above the cutoff but are not in a methylated motif or a secondary peak.
} 


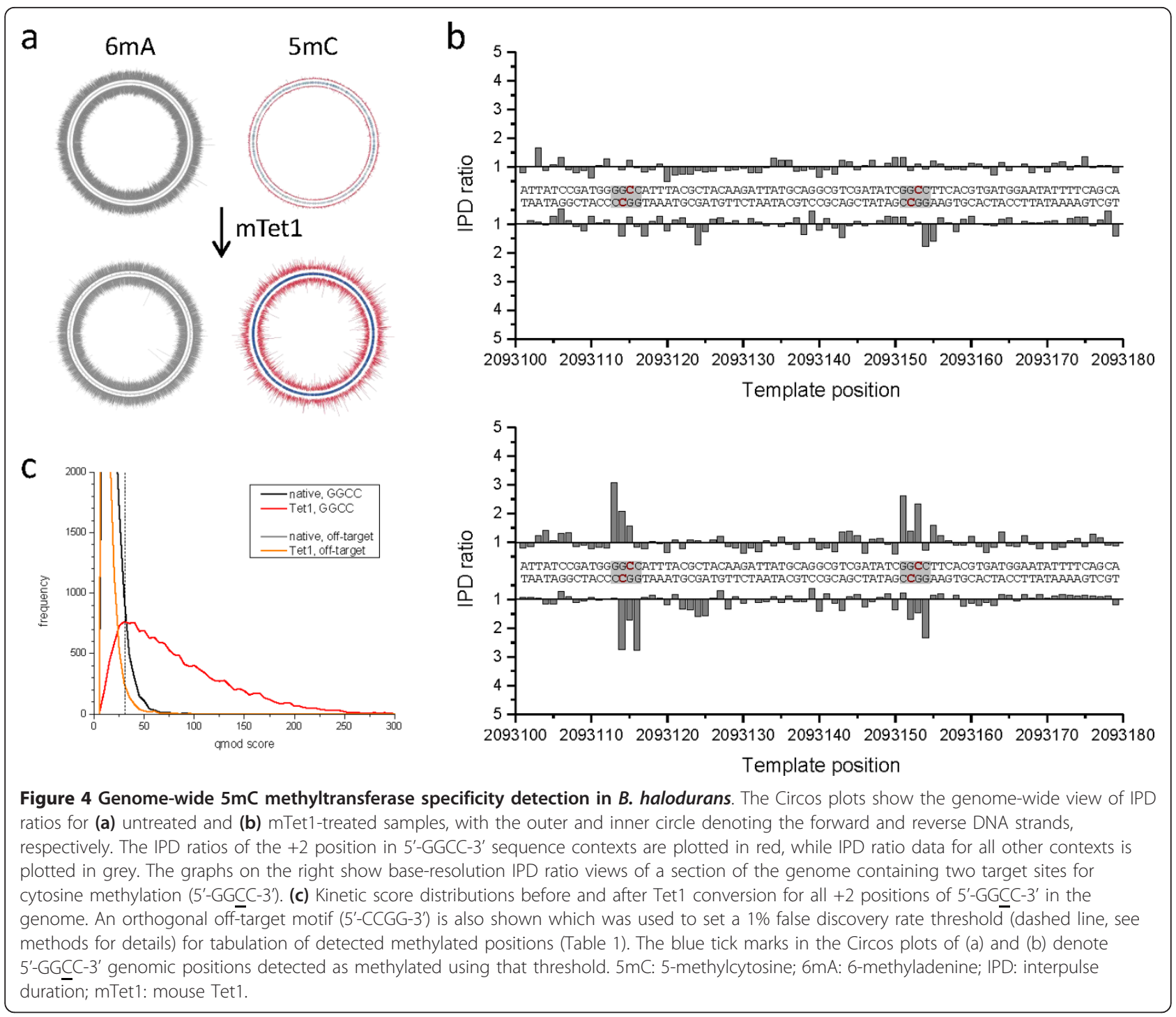

demonstrated through bulk biochemical and genetic studies that the dynamics of methylation in bacteria plays critical roles in basic cellular functions as well as directly affecting virulence [11,12,27].

The kinetic signatures of $4 \mathrm{mC}$ and $5 \mathrm{caC}$ are sufficiently different to allow for discrimination of the two types of cytosine modifications in bacteria. When sequencing through $4 \mathrm{mC}$, the polymerase slows down only when incorporating the cognate nucleotide opposite the modification, with no significant secondary IPD ratio peaks [16]. By contrast, the primary IPD ratio peak for $5 \mathrm{caC}$ is located two bases after the modification $(+2$ position). The combination of observing the sequence identity and the specific kinetic signature make it possible to not only discover the presence of a base modification but also to determine the chemical identity of the type of modification. We are working on algorithmically harnessing this information contained in the kinetic signatures to expand the power of direct detection of modified bases unique to SMRT sequencing [28]. Algorithms that incorporate IPD data from multiple positions across the entire footprint of the polymerase may further enhance the ability to detect and discriminate between modification types. This multi-site analysis and a further understanding of the sequence context dependence of the $5 \mathrm{caC}$ kinetic signature should improve detection of $5 \mathrm{caC}$, potentially reducing the sequencing coverage needed to detect converted $5 \mathrm{mC}$ positions even further.

In higher eukaryotes, the epigenome is much more complex as at least four different forms of cytosine can occur and dynamically interconvert at epigenetically regulated genomic positions. Emerging evidence suggests that the Tet proteins and the modified cytosines they generate are crucial for a growing list of biological 
processes, including zygotic epigenetic reprogramming, pluripotent stem cell differentiation, hematopoiesis and development of leukemia [2]. Thus, methods for comprehensive genome-wide mapping of all cytosine modifications will be critical for epigenomic studies. Several methods have been described recently for discriminating between $5 \mathrm{mC}$ and $5 \mathrm{hmC}$ using bisulfite sequencing in combination with chemical or enzymatic conversion $[14,15]$. Since for a given sequence context in SMRT sequencing, the kinetic signatures of $5 \mathrm{mC}, 5 \mathrm{hmC}, 5 \mathrm{fC}$, and $5 \mathrm{caC}$ are different, there is the potential for direct identification of the various modifications on native DNA samples. We are working to expand the bioinformatics analysis algorithms towards discrimination of different epigenetic marks, taking into account the different signatures as a function of sequence context, as well as partial modification and mixtures of modification types. There are already several strategies for enhancing the kinetic signature of two cytosine modifications allowing for direct detection of $5 \mathrm{mC}$ and $5 \mathrm{hmC}$ in a single sample using SMRT sequencing. $5 \mathrm{hmC}$ positions can first be glucosylated [21], followed by Tet1-mediated oxidation of $5 \mathrm{mC}$ to $5 \mathrm{caC}$. Glucosylated $5 \mathrm{hmC}$ will be protected from conversion and discrimination of the two forms can be made based on the differing kinetic signatures. We expect that these and further advances in the direct detection of modified bases during routine genome sequencing will become an important tool to further our understanding of genome and epigenome function.

\section{Methods \\ Materials}

Custom oligonucleotides containing modified bases were synthesized on-site or purchased from Trilink BioTechnologies (San Diego, CA, USA) and Integrated DNA Technologies (Coralville, IA, USA). All oligonucleotides contained 5' phosphate groups. The plasmid (pCRBlunt) was obtained from Life Technologies (Carlsbad, CA, USA). A list of the sequences can be found in Additional file 6.

Bacterial strains and/or genomic DNA from bacterial strains were purchased from the American Type Culture Collection (Manassas, VA, USA). The following strains were used in this study: E. coli K12 MG1655, and B. halodurans C-125 (JCM 9153).

\section{SMRTbell template preparation}

Synthetic SMRTbell templates were made as previously described by ligating several synthetic oligonucleotides [20]. For plasmid and genomic DNA samples, an aliquot of approximately $25 \mathrm{ng}$ of DNA was subjected to WGA using the REPLI-g Midi Kit (Qiagen, Valencia, CA, USA). WGA and native DNA was sheared to an average size of approximately 500 bp via adaptive focused acoustics (Covaris, Woburn, MA, USA). SMRTbell template sequencing libraries were prepared as previously described $[16,29]$. SMRTbell libraries made from wholegenome-amplified pCRBlunt-6K plasmid were in vitro methylated using the HpaII MTase (recognition sequence: 5'-C ${ }^{5 \mathrm{~m}}$ CGG-3'; New England BioLabs; Ipswich, MA, USA) as per the manufacturer's instructions. Complete methylation was assessed by modifying lambda DNA in parallel and subjecting to methylationsensitive restriction using the HpaII restriction enzyme (New England BioLabs).

\section{Tet1 conversion}

The $5 \mathrm{mC}$ modifications in SMRTbell template libraries were converted to $5 \mathrm{caC}$ using the $5 \mathrm{mC}$ mTet1 Oxidation Kit from Wisegene (Chicago, IL, USA) as per the manufacturer's instructions. Approximately $500 \mathrm{ng}$ of SMRTbell templates were treated with the Tet 1 enzyme at $37^{\circ} \mathrm{C}$ for 60 minutes followed by proteinase $\mathrm{K}$ treatment at $50^{\circ} \mathrm{C}$ for 60 minutes. Converted SMRTbell templates were purified using Micro Bio-Spin 30 Columns (BioRad, Hercules, CA, USA) with additional purification and concentration using MinElute PCR Purification Columns (Qiagen).

\section{Sequencing and data acquisition}

SMRTbell templates were subjected to standard SMRT sequencing, as described $[18,19]$. Reads were processed and mapped to the respective reference sequences using the BLASR mapper [30] and Pacific Biosciences' SMRT Analysis pipeline [31] using the standard mapping protocol. IPDs were measured as previously described [17] and processed as described [16] for all pulses aligned to each position in the reference sequence.

For the bacterial methylome analysis [10], we used Pacific Biosciences' SMRTPortal analysis platform v. 1.3.1, which uses an in silico kinetic reference and a $t$-test based detection of modified base positions [32]. The following GenBank reference sequences were used: U00096.2 for E. coli K-12 MG1655 and BA000004.3 for $B$. halodurans $\mathrm{C}-125$. MTase target sequence motifs were identified by selecting the top 1,000 kinetic hits and subjecting a \pm 20 base window around the detected base to MEME-ChIP [33], and compared to the predictions in REBASE [24]. To estimate the enhancement of detection of methylated $5 \mathrm{mC}$ positions (Table 1), we first selected an orthogonal off-target motif of similar sequence content and calculated the kinetic score representing the $99^{\text {th }}$ percentile of all genomic positions of that motif (5'GGWCC-3' for E. coli (score threshold = 35.6); 5'CCGG-3' for B. halodurans (30.4)). We then used this $1 \%$ false positive detection threshold for determining the number of genomic positions of the on-target methylation sites detected as methylated (Figures $3 \mathrm{c}$ and 4c; 
Table 1). IPD ratio plots were visualized using Circos [34].

\section{Additional data files}

The following additional data are available with the online version of the paper. Additional data file 1 is a figure that demonstrates the sequence context dependence of the kinetic signatures for $5 \mathrm{mC}$ and $5 \mathrm{caC}$. Additional data file 2 is a figure that shows IPD ratio data for synthetic SMRTbell templates before and after conversion of $5 \mathrm{mC}$ to $5 \mathrm{caC}$. Additional file 3 is a figure with IPD ratio distributions for all methylated sequence motifs in E. coli and B. halodurans. Additional files 4 and 5 are tables that contain detection rate information for all methylated motifs in E. coli and B. halodurans, respectively. Additional data file 6 is a table of oligonucleotide sequences used in this study.

\section{Additional material}

Additional file 1: Sequence context dependence of the kinetic signatures for $5 \mathrm{mC}$ and $\mathbf{5 c a C}$. Top panel (a) is a schematic of the synthetic SMRTbell template with random bases surrounding $5 \mathrm{mC}$ or $5 \mathrm{caC}$ modifications in a CG sequence context. The modified position is indicated with pink text and an asterisk. The bottom panel (b) is a heat map of IPD ratio values of either $5 \mathrm{mC}$ or $5 \mathrm{caC}$ relative to an unmodified control sequence. IPD ratio values are shown for all possible sequence contexts of four random bases over ten positions on the DNA template ( -3 to +6 relative to the modified base). Light grey boxes within the heatmap denote sequence contexts that did not have sufficient sequencing coverage. A minimum of 10 independent molecules of both modified and control templates were analyzed.

Additional file 2: Conversion of $5 \mathrm{mC}$ to $5 \mathrm{caC}$ in synthetic oligonucleotides. Kinetic signals for synthetic oligonucleotides carrying two $5 \mathrm{mC}$ modified sites (red bars) are shown before (top) and after (bottom) mTet1-mediated oxidation to $5 \mathrm{caC}$. IPD ratio data are plotted for each template position relative to a control template of identical sequence but lacking modifications. The template is shown in the $5^{\prime}$ to $3^{\prime}$ direction from left to right, the polymerase movement is right to left across the template as indicated by the arrow.

Additional file 3: IPD ratio distributions of all methylated motifs in E.coli MG1655 and B.halodurans C-125. Each plots show the histograms of IPD ratio values for each methylated motif and an offtarget non-methylated motif. The top plots are from native samples and the bottom show the same data after Tet1-mediated conversion of $5 \mathrm{mC}$ to $5 \mathrm{caC}$.

Additional file 4: Table of detection rates for all methylated motifs in E.coli MG1655. The number and percent detection is shown for all methylated sequence motifs in the genome. A detected genomic position is one that has a kinetic score that is greater than the cutoff value. Detection rates are also shown for common secondary IPD ratio peaks of $6 \mathrm{~mA}(+5)$ and $5 \mathrm{mC}(+2,+6)$ and for off-target motifs with similar sequence content to the methylated motifs. Methylated bases are colored: $6 \mathrm{~mA}$ (red), $5 \mathrm{mC}$ (blue). The interrogated base in the motif is underlined. Unassigned are genomic positions with kinetic scores above the cutoff which are not in a methylated motif or a secondary peak.

Additional file 5: Table of detection rates for all methylated motifs in B.halodurans C-125. The number and percent detection is shown for all methylated sequence motifs in the genome. A detected genomic position is one that has a kinetic score that is greater than the cutoff value. Detection rates are also shown for common secondary IPD ratio peaks of $6 \mathrm{~mA}(+5)$ and $5 \mathrm{mC}(+2,+6)$ and for off-target motifs with similar sequence content to the methylated motifs. Methylated bases are colored: $6 \mathrm{~mA}$ (red), $5 \mathrm{mC}$ (blue). The interrogated base in the motif is underlined. Unassigned are genomic positions with kinetic scores above the cutoff which are not in a methylated motif or a secondary peak.

Additional file 6: Table of oligonucleotide sequences used in this study.

\section{Abbreviations}

4mC: 4-methylcytosine; 5caC: 5-carboxylcytosine; 5fC: 5-formylcytosine; 5hmC: 5-hydroxymethylcytosine; $5 \mathrm{mC}: 5$-methylcytosine; $6 \mathrm{~mA}: 6$ methyladenine; bp: base pair; IPD: interpulse duration; MTase: methyltransferase; mTet1: mouse Tet1; SMRT: single-molecule: real-time; Tet Ten-eleven translocation; WGA: whole genome amplification.

\section{Authors' contributions}

$\mathrm{TC}, \mathrm{ST}, \mathrm{CH}$, and JK conceived of the study. TC, XL, and QD carried out mTet1 conversions. TC and MB generated sequencing libraries and carried out the sequencing reactions. TC, KL, and JK carried out data analysis. TC and JK made figures and wrote the manuscript. All authors read and approved the final manuscript

\section{Competing interests}

$T C, K L, M B, S T$, and JK are full-time employees at Pacific Biosciences, a company commercializing single-molecule, real-time nucleic acid sequencing technologies. The University of Chicago Office of Technology and Intellectual Property has filed patent application for the $5 \mathrm{mC}$-oxidation strategy.

\section{Acknowledgements}

We thank S. Kamtekar, K. Spittle, J. Londry, and P. Marks for helpful discussions, assistance with sample preparation and data analysis, and Min Zhou for generously providing mTet1 conversion kits.

\section{Author details}

'Pacific Biosciences, 1380 Willow Road, Menlo Park, CA 94025, USA. ${ }^{2}$ Department of Chemistry and Institute for Biophysical Dynamics, 929 East 57th Street, The University of Chicago, Chicago, Illinois, 60637, USA.

Received: 24 July 2012 Accepted: 22 January 2013

Published: 22 January 2013

\section{References}

1. Bird A: DNA methylation patterns and epigenetic memory. Genes Dev 2002, 16:6-21.

2. Wu H, Zhang Y: Mechanisms and functions of Tet protein-mediated 5-methylcytosine oxidation. Genes Dev 2011, 25:2436-2452.

3. Kriaucionis $\mathrm{S}$, Heintz N: The nuclear DNA base 5-hydroxymethylcytosine is present in Purkinje neurons and the brain. Science 2009, 324:929-930.

4. Tahiliani M, Koh KP, Shen Y, Pastor WA, Bandukwala H, Brudno Y, Agarwal S, lyer LM, Liu DR, Aravind L, Rao A: Conversion of 5-methylcytosine to 5-hydroxymethylcytosine in mammalian DNA by MLL partner TET1. Science 2009, 324:930-935.

5. Pfaffeneder T, Hackner B, Truss M, Munzel M, Muller M, Deiml CA, Hagemeier C, Carell T: The discovery of 5-formylcytosine in embryonic stem cell DNA. Angew Chem Int Ed Engl 2011, 50:7008-7012.

6. He YF, Li BZ, Li Z, Liu P, Wang Y, Tang Q, Ding J, Jia Y, Chen Z, Li L, Sun Y, Li X, Dai Q, Song CX, Zhang K, He C, Xu GL: Tet-mediated formation of 5-carboxylcytosine and its excision by TDG in mammalian DNA. Science 2011, 333:1303-1307.

7. Ito S, Shen L, Dai Q, Wu SC, Collins LB, Swenberg JA, He C, Zhang Y: Tet proteins can convert 5-methylcytosine to 5-formylcytosine and 5-carboxylcytosine. Science 2011, 333:1300-1303.

8. Zhang L, Lu X, Lu J, Liang H, Dai Q, Xu G-L, Luo C, Jiang H, He C: Thymine DNA glycosylase specifically recognizes 5-carboxylcytosine-modified DNA. Nat Chem Biol 2012, 8:328-330.

9. Münzel M, Globisch D, Carell T: 5-hydroxymethylcytosine, the sixth base of the genome. Angew Chem Int Ed Engl 2011, 50:6460-6468. 
10. Murray IA, Clark TA, Morgan RD, Boitano M, Anton BP, Luong K, Fomenkov A, Turner SW, Korlach J, Roberts RJ: The methylomes of six bacteria. Nucleic Acids Res 2012, 40:11450-11462.

11. Marinus MG, Casadesus J: Roles of DNA adenine methylation in hostpathogen interactions: mismatch repair, transcriptional regulation, and more. FEMS Microbiol Rev 2009, 33:488-503.

12. Low DA, Weyand NJ, Mahan MJ: Roles of DNA adenine methylation in regulating bacterial gene expression and virulence. Infect Immun 2001, 69:7197-7204.

13. Huang $Y$, Pastor WA, Shen Y, Tahiliani M, Liu DR, Rao A: The behaviour of 5-hydroxymethylcytosine in bisulfite sequencing. PloS One 2010, 5:e8888.

14. Yu M, Hon GC, Szulwach KE, Song CX, Zhang L, Kim A, Li X, Dai Q, Shen Y, Park B, Min JH, Jin P, Ren B, He C: Base-resolution analysis of 5-hydroxymethylcytosine in the mammalian genome. Cell 2012, 149:1368-1380.

15. Booth MJ, Branco MR, Ficz G, Oxley D, Krueger F, Reik W, Balasubramanian S: Quantitative sequencing of 5-methylcytosine and 5-hydroxymethylcytosine at single-base resolution. Science 2012, 336:934-937.

16. Clark TA, Murray IA, Morgan RD, Kislyuk AO, Spittle KE, Boitano M, Fomenkov A, Roberts RJ, Korlach J: Characterization of DNA methyltransferase specificities using single-molecule, real-time DNA sequencing. Nucleic Acids Res 2012, 40:e29.

17. Flusberg BA, Webster DR, Lee JH, Travers KJ, Olivares EC, Clark TA, Korlach J, Turner SW: Direct detection of DNA methylation during single-molecule, real-time sequencing. Nat Methods 2010, 7:461-465.

18. Eid J, Fehr A, Gray J, Luong K, Lyle J, Otto G, Peluso P, Rank D, Baybayan P, Bettman B, Bibillo A, Bjornson K, Chaudhuri B, Christians F, Cicero R, Clark S, Dalal R, Dewinter A, Dixon J, Foquet M, Gaertner A, Hardenbol P, Heiner C, Hester K, Holden D, Kearns G, Kong X, Kuse R, Lacroix Y, Lin S, Lundquist P, et al: Real-time DNA sequencing from single polymerase molecules. Science 2009, 323:133-138.

19. Korlach J, Bjornson KP, Chaudhuri BP, Cicero RL, Flusberg BA, Gray JJ, Holden D, Saxena R, Wegener J, Turner SW: Real-time DNA sequencing from single polymerase molecules. Methods Enzymol 2010, 472:431-455.

20. Clark TA, Spittle KE, Turner SW, Korlach J: Direct detection and sequencing of damaged DNA bases. Genome Integr 2011, 2:10.

21. Song CX, Clark TA, Lu XY, Kislyuk A, Dai Q, Turner SW, He C, Korlach J: Sensitive and specific single-molecule sequencing of 5hydroxymethylcytosine. Nat Methods 2012, 9:75-77.

22. Kamtekar S, Berman AJ, Wang J, Lazaro JM, de Vega M, Blanco L, Salas M, Steitz TA: Insights into strand displacement and processivity from the crystal structure of the protein-primed DNA polymerase of bacteriophage phi29. Mol Cell 2004, 16:609-618.

23. Wilson GG, Murray NE: Restriction and modification systems. Ann Rev Genet 1991, 25:585-627.

24. Roberts RJ, Vincze T, Posfai J, Macelis D: REBASE-a database for DNA restriction and modification: enzymes, genes and genomes. Nucleic Acids Res 2010, 38:D234-236.

25. Bashir A, Klammer AA, Robins WP, Chin C-S, Webster D, Paxinos E, Hsu D, Ashby M, Wang S, Peluso P, Sebra R, Sorenson J, Bullard J, Yen J, Valdovino M, Mollova E, Luong K, Lin S, LaMay B, Joshi A, Rowe L, Frace M, Tarr CL, Turnsek M, Davis BM, Kasarskis A, Mekalanos JJ, Waldor MK, Schadt EE: A hybrid approach for the automated finishing of bacterial genomes. Nat Biotech 2012, 30:701-707.

26. Koren S, Schatz MC, Walenz BP, Martin J, Howard JT, Ganapathy G, Wang Z, Rasko DA, McCombie WR, Jarvis ED, Phillippy AM: Hybrid error correction and de novo assembly of single-molecule sequencing reads. Nat Biotech 2012, 30:6.93-700.

27. Fang G, Munera D, Friedman DI, Mandlik A, Chao MC, Banerjee O, Feng Z, Losic B, Mahajan MC, Jabado OJ, Deikus G, Clark TA, Luong K, Murray IA, Davis BM, Keren-Paz A, Chess A, Roberts RJ, Korlach J, Turner SW, Kumar V, Waldor MK, Schadt EE: Genome-wide mapping of methylated adenine residues in pathogenic Escherichia coli using single-molecule real-time sequencing. Nature Biotechnol 2012, 30:1232-1239.

28. Schadt E, Banerjee O, Fang G, Feng Z, Wong W, Zhang X, Kislyuk A, Clark T, Luong K, Keren-Paz A, Chess A, Kumar V, Chen-Plotkin A, Sondheimer N, Korlach J, Kasarskis A: Modeling kinetic rate variation in third generation DNA sequencing data to detect putative modifications to DNA bases. Genome Res 2012, 23:129-141.
29. Travers KJ, Chin CS, Rank DR, Eid JS, Turner SW: A flexible and efficient template format for circular consensus sequencing and SNP detection. Nucleic Acids Res 2010, 38:e159.

30. BLASR. [http://www.smrtcommunity.com/SMRT-Analysis/Algorithms/BLASR].

31. SMRT Analysis. [http://www.smrtcommunity.com/SMRT-Analysis/Software/ SMRT-Analysis].

32. Detecting DNA Base Modifications. [http://www.pacb.com/pdf/ TN_Detecting_DNA_Base_Modifications.pdf].

33. Machanick P, Bailey TL: MEME-ChIP: motif analysis of large DNA datasets. Bioinformatics 2011, 27:1696-1697.

34. Krzywinski M, Schein J, Birol I, Connors J, Gascoyne R, Horsman D, Jones SJ, Marra MA: Circos: an information aesthetic for comparative genomics. Genome Res 2009, 19:1639-1645.

doi:10.1186/1741-7007-11-4

Cite this article as: Clark et al:: Enhanced 5-methylcytosine detection in single-molecule, real-time sequencing via Tet1 oxidation. BMC Biology 2013 11:4.

\section{Submit your next manuscript to BioMed Central and take full advantage of:}

- Convenient online submission

- Thorough peer review

- No space constraints or color figure charges

- Immediate publication on acceptance

- Inclusion in PubMed, CAS, Scopus and Google Scholar

- Research which is freely available for redistribution 